\title{
Cricopharyngeal peroral endoscopic myotomy improves oropharyngeal dysphagia in patients with Parkinson's disease $\square$
}

다 (i) $(-)$

\section{Authors}

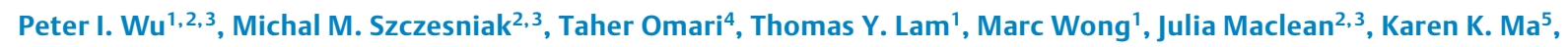
Anne Y. Chan ${ }^{5}$, Vincent Mok ${ }^{5}$, Ian J. Cook ${ }^{2,3}$, Charles Cock ${ }^{4}$, Joseph Sung ${ }^{1}$, Justin Wu ${ }^{1}$, Philip W. Chiu ${ }^{1}$

Institutions

1 Institute of Digestive Disease, Prince of Wales Hospital, Chinese University of Hong Kong, HKSAR, China

2 Department of Gastroenterology and Hepatology, St George Hospital, Sydney, Australia

3 St George and Sutherland Clinical School, University of NSW, Sydney, Australia

4 College of Medicine and Public Health, Flinders University, Adelaide, Australia

5 Margaret K. L. Cheung Research Centre for Management of Parkinsonism, Division of Neurology, Prince of Wales Hospital, Chinese University of Hong Kong, HKSAR, China

\section{Bibliography}

Endosc Int Open 2021; 09: E1811-E1819

DOI 10.1055/a-1562-7107

ISSN 2364-3722

(C) 2021. The Author(s).

This is an open access article published by Thieme under the terms of the Creative Commons Attribution-NonDerivative-NonCommercial License, permitting copying and reproduction so long as the original work is given appropriate credit. Contents may not be used for commercial purposes, or adapted, remixed, transformed or built upon. (https://creativecommons.org/licenses/by-nc-nd/4.0/)

Georg Thieme Verlag KG, Rüdigerstraße 14,

70469 Stuttgart, Germany

\section{Corresponding author}

Dr. Peter I. Wu, Department of Gastroenterology and

Hepatology, St. George Hospital, Sydney, NSW, Australia 2217

Fax: +91133993

p.wu@unsw.edu.au

\section{ABSTRACT}

Background and study aims Oropharyngeal dysphagia (OPD) is prevalent in patients with Parkinson's disease (PD). Upper esophageal sphincter (UES) dysfunction is an important pathophysiological factor for OPD in PD. The cricopharyngeus (CP) is the main component of UES. We assessed the preliminary efficacy of cricopharyngeal peroral endoscopic myotomy (C-POEM) as a treatment for dysphagia due to UES dysfunction in PD.

Patients and methods Consecutive dysphagic PD patients with UES dysfunction underwent C-POEM. Swallow metrics derived using high-resolution pharyngeal impedance manometry (HRPIM) including raised UES integrated relaxation pressure (IRP), raised hypopharyngeal intrabolus pressure (IBP), reduced UES opening caliber and relaxation time defined UES dysfunction. Sydney Swallow Questionnaire (SSQ) and Swallowing Quality of Life Questionnaire (SWAL-QOL) at before and 1 month after C-POEM measured symptomatic improvement in swallow function. HRPIM was repeated at 1-month follow-up.

Results C-POEM was performed without complications in all $(n=8)$ patients. At 1 month, there was an improvement in both the mean SSQ (from 621.5 to 341.8 , mean difference $-277.3,95 \% \mathrm{Cl}[-497.8,-56.7], P=0.02)$ and SWALQOL (from 54.9 to 68.3 , mean difference $9.1,95 \% \mathrm{Cl}[0.7$, 17.5], $P=0.037$ ) scores. Repeat HRPIM confirmed a decrease in both the mean UES IRP $(13.7 \mathrm{~mm} \mathrm{Hg}$ to $3.6 \mathrm{~mm}$ $\mathrm{Hg}$, mean difference $-10.1 \mathrm{~mm} \mathrm{Hg}, 95 \% \mathrm{Cl}[-16.3,-3.9], P$ $=0.007)$ and the mean hypopharyngeal IBP $(23.5 \mathrm{~mm} \mathrm{Hg}$ to $10.4 \mathrm{~mm} \mathrm{Hg}$, mean difference $-11.3 \mathrm{~mm} \mathrm{Hg}, 95 \% \mathrm{Cl}[-17.2$, -5.4], $P=0.003)$.

Conclusions In dysphagic PD patients with UES dysfunction, C-POEM is feasible and enhances UES relaxation and reduces sphincteric resistance to flow during the swallow, thereby improving dysphagia symptoms. 


\section{Introduction}

Parkinson's disease (PD) is the second most prevalent neurodegenerative disorder after Alzheimer's disease [1]. The prevalence of PD (per 100,000) increases exponentially with age, from 41 in individuals aged 40 to 49 years to approximately 1900 in individuals over 80 years old [2]. The prevalence of PD is also increasing with time, with an estimate of 9 million individuals with PD globally by the year 2030 [3]. Oropharyngeal dysphagia (OPD) affects up to $80 \%$ of patients with PD [4] and is often unresponsive to L-Dopa treatment [5]. This means that the dysphagia burden attributable to UES dysfunction is significant, supporting a need for an effective treatment option.

Manometric [6] and electrophysiological [7] studies have identified impaired relaxation of the upper esophageal sphincter (UES) in at least $20 \%$ to $30 \%$ of dysphagic PD patients, as a major pathophysiological factor underpinning dysphagia. The cricopharyngeus $(C P)$ is the major muscular component of the UES. Transcutaneous CP myotomy has been shown to be an effective treatment in some cases [8-10] and yielded an overall response rate of $63 \%$ [9] in one uncontrolled series. However, efficacy data are limited to small retrospective series. Moreover, the associated mortality and morbidity makes the transcutaneous approach an unattractive treatment option for a disease with a very high prevalence in the elderly. In a large series of 253 patients undergoing CP myotomy, the postoperative mortality rate was $1.6 \%$ and serious adverse events (including permanent tracheostomy, infection, and nerve injury) occurred in as up to $15 \%$ of the cases [11].

Peroral endoscopic myotomy (POEM) is a minimally invasive endoscopic technique developed to treat esophageal achalasia [12]. The indications for this technique have since expanded beyond its original intention, including non-achalasia spastic esophageal disorders and Zenker's diverticulum, and pyloromyotomy for gastroparesis [13-16]. The advent of "thirdspace" endoscopy presents a unique, potentially less invasive, opportunity to advance the treatment of OPD in PD patients. At the very least, it is biologically plausible that such endoscopic technique can be an effective treatment for symptomatic proven UES dysfunction.

We adapted the endoscopic principles of POEM to the CP (CPOEM) intended as a treatment of UES dysfunction contributing to dysphagia in cases of PD. We aimed to demonstrate the proof of principle by assessing: 1) the feasibility and safety; 2) objective biomechanical changes including manometric, radiographic, and distensibility measurements; and 3) preliminary efficacy data.

\section{Patients and methods}

\section{Study protocol}

Between July 15, 2019 and January 31, 2021, a total of 11 consecutive PD patients with self-reported OPD symptoms and manometric evidence of UES dysfunction (see below) were invited to undergo C-POEM. Validated self-reported questionnaires, Sydney Swallow Questionnaire (SSQ) $[17,18]$ and Swallow Quality of Life Questionnaire (SWAL-QOL) $[19,20]$ assessed the severity of dysphagia symptoms and dysphagia-specific quality of life (QoL), respectively. Other pre-operative assessment included a videofluoroscopic swallow study (VFSS) to measure radiographic opening of the UES during swallowing (see below). During C-POEM, the CP distensibility was measured using Functional Luminal Imaging Probe (FLIP).

At 1-month follow-up, repeat HRPIM and VFSS were performed to objectively evaluate potential myotomy-induced biomechanical changes in the UES function. Patients were also asked to complete the questionnaires (SSQ and SWAL-QOL) during clinical reviews. Institutional Review Board ethical approval was obtained for the study (Joint Chinese University of Hong Kong-New Territories East Cluster Clinical Research Ethics Committee, NTEC 2019.183). This study was prospectively registered at the Australian New Zealand Clinical Trial Registry (ACTRN12619000927123).

\section{High-resolution pharyngeal impedance manometry}

Data on high-resolution pharyngeal impedance manometry (HRPIM) were collected using a 3.2-mm diameter catheter (36 solid-state pressure sensors at $1 \mathrm{~cm}, 12,2$-cm impedance segments, Unisensor/Laborie, Switzerland). The catheter was positioned with pressure sensors spanning from velopharynx to proximal esophagus. With the patient sitting upright in a neutral head position, a triplicate swallow of $10-\mathrm{mL}$ thin saline liquids (International Diet Dysphagia Standardization Initiative 0; SBM Kit, Tricso, Australia) was performed. Four core HRPIM metrics pertaining UES functions were derived [21] ( 1) UES Integrated Relaxation Pressure (IRP) quantifies the lowest non-consecutive 0.25 seconds of UES relaxation during deglutition; 2) Hypopharyngeal Intrabolus Pressure (IBP) measures (indirectly) the UES restriction to bolus flow; 3) UES Maximal Admittance (MaxAd) is a surrogate marker for maximal deglutitive luminal cross-sectional area; and 4) UES relaxation time (RT) measures the deglutitive UES relaxation duration. Abnormality was defined as values outside of $95 \%$ reference range interval established from historical laboratory controls studied using identical equipment [22].

UES dysfunction, for the purpose of the study, was defined as impaired UES relaxation (i. e., IRP $>2.9 \mathrm{~mm} \mathrm{Hg}$ ) [22], and the presence of one or more of the following measures of the biomechanical consequences of impaired UES relaxation: increased trans-sphincteric resistance (i.e. raised IBP); reduced UES opening diameter (i.e. reduced MaxAd), or reduced UES opening duration (i. e. reduced RT) during 10-mL thin liquid bolus swallows.

Immediately after the HRPIM was complete, the catheter was repositioned distally into the proximal stomach, with pressure sensors spanning across the lower esophageal sphincter. With patients in a supine position, a total of 10 swallows of 5 $\mathrm{mL}$ thin saline liquids was performed to exclude achalasia of the cardia. 

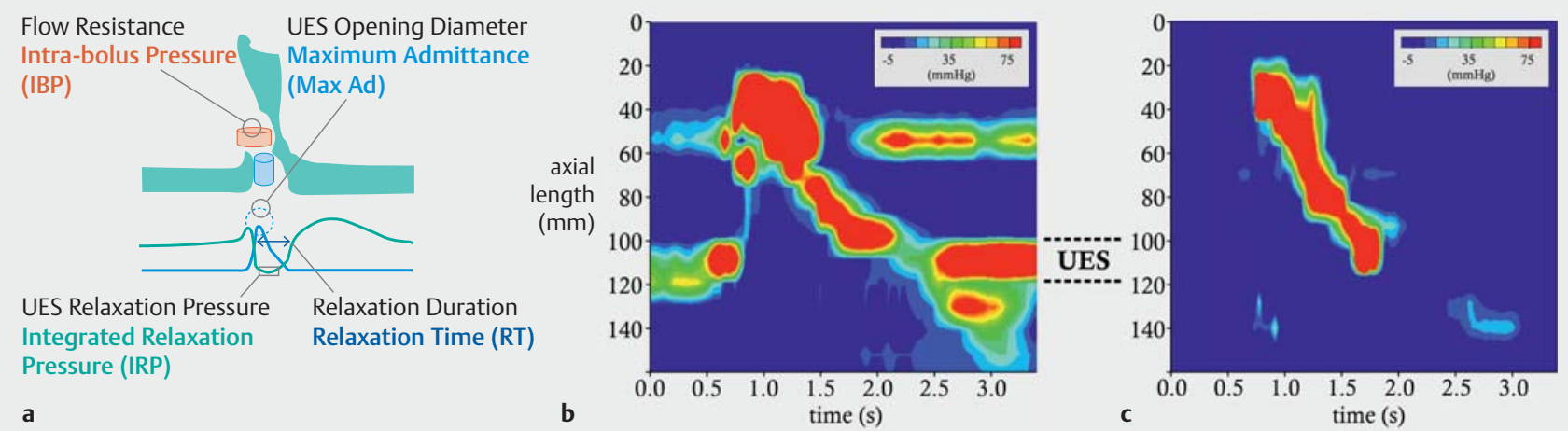

- Fig. 1 HRPIM example of a 10-mL thin liquid swallow in a dysphagic PD patient who underwent C-POEM. a Schematic of a swallow event illustrating the key metrics derived from the concurrent pressure (green) and impedance (impedance) recordings using e-sleeve across the UES. b Before C-POEM, UES basal tone can be easily identified as a horizontal high-pressure band that relaxes at the onset of swallow event. c After C-POEM, the UES basal tone became much harder to identify, the pharyngeal contractility was however preserved.

\section{Intraprocedural cricopharyngeal distensibility measurements}

The potential myotomy-induced changes in the intrinsic CP distensibility were measured using FLIP immediately pre- and post- C-POEM during the procedure under anesthesia. The measurement technique had been described previously [23, 24]. Briefly, an 8-cm catheter (EF-325N, EndoFLIP, Medtronic, United States) was passed trans-orally into the esophageal lumen with the cylindrical bag straddling the CP. To avoid unintentional tissue injuries during insertion, the catheter was passed over a guidewire through suture ties placed along the non-functioning parts of the catheter [23]. The bag was infused from $0 \mathrm{~mL}$ to $40 \mathrm{~mL}$ at a rate of $60 \mathrm{~mL} / \mathrm{min}$. During distension, concurrent 16 cross-sectional areas and intra-bag pressure were recorded from which the CP distensibility was derived. The CP distensibility index (CP-DI) was defined as a function of narrowest cross-sectional area (nCSA) over the corresponding intra-bag pressure at a distension volume of $40 \mathrm{~mL}$, averaged over a 30-second period of contractile inactivity.

\section{Videofluoroscopic swallow study}

A radio-opaque scale was placed on each patient's neck in the field of view to calculate a magnification factor to determine accurate lumen diameters. Patients were administered triplicates of $10 \mathrm{ml}$ of barium suspension (E-Z-PAQUE, Bracco Diagnostics) in both sagittal and anteroposterior projections. Radiographic cineloops were acquired at 25 frames per second and digitized. Subsequently they were imported into Imagej (version $1.53 \mathrm{~h}$ ) software and the second $10-\mathrm{mL}$ swallow in the sagittal plane was analyzed from each study. Firstly, the scale visible in the radiographic image was used to derive a pixel/mm ratio to enable conversion of measurements made on the images to millimeters. Then the UES dimensions during sphincter opening were measured frame-by-frame at temporal resolution of 0.04 seconds. From this analysis we could obtain the duration as well as the maximum UES opening. During a swallow sequence bolus flow across the UES is influenced by both the diameter and duration of the opening. To account for this, we cal- culated area under the diameter vs time curve for each of the swallows (AUC).

\section{C-POEM (video)}

The CP is a "C"-shaped striated muscle band which attaches to the lateral margins of the cricoid cartilage anteriorly. It is bordered superiorly by the inferior pharyngeal constrictor and merges inferiorly with the longitudinal, striated muscle of the cervical esophagus. To avoid recurrent laryngeal nerve injuries, the C-POEM is performed via a posterior approach with the patient in a left lateral position under general anesthesia. Equipment includes a cap-fitted (Space Adjustor, Top, Japan, or ST Hood, Fujifilm, Japan) adult gastroscope (GIF-H290Z, Olympus, Japan), $\mathrm{CO}_{2}$ insufflation, and electric surgical Triangle-Tip Knife (KD-640 L, Olympus, Japan). An initial submucosal bleb was followed by a longitudinal $1-2 \mathrm{~cm}$ length mucosotomy in the hypopharynx commencing 2- to 3-cm cranial to the endoscopically identified upper margin of the CP ( $\triangleright$ Fig. 2). Next, a short (5to $6-\mathrm{cm}$ ) submucosal tunnel is created extending into the proximal esophageal wall thereby exposing the horizontal fibers of the CP. Full-thickness dissection of the CP (3- to 4-cm in length) is then performed using the electric surgical knife. Hemostasis was achieved using either coagulating forceps (Coagrasper, FD$411 \mathrm{QR}$, Olympus, Japan) or the Triangle-Tip Knife. Lastly, between five and seven through-the-scope endoscopic clips (Resolution Clip, Boston Scientific, USA, or SureClip, Micro-Tech, China) were placed to close the hypopharyngeal mucosotomy. Postoperatively, patients receive 24 hours of prophylactic intravenous co-amoxiclav and analgesia on an as required basis. (See example video $>$ Video 1.)

\section{Data and statistical analysis}

De-identified HRPIM data were exported to Swallow Gateway (SwallowGateway.com, Flinders University, Australia), an online application for analysis. The analysis was conducted by authors PW and MS and cross-checked by authors TO and CC. CP distensibility data were exported and analyzed using MATLAB (The Mathworks Inc., Natick, Massachusetts, United States, 


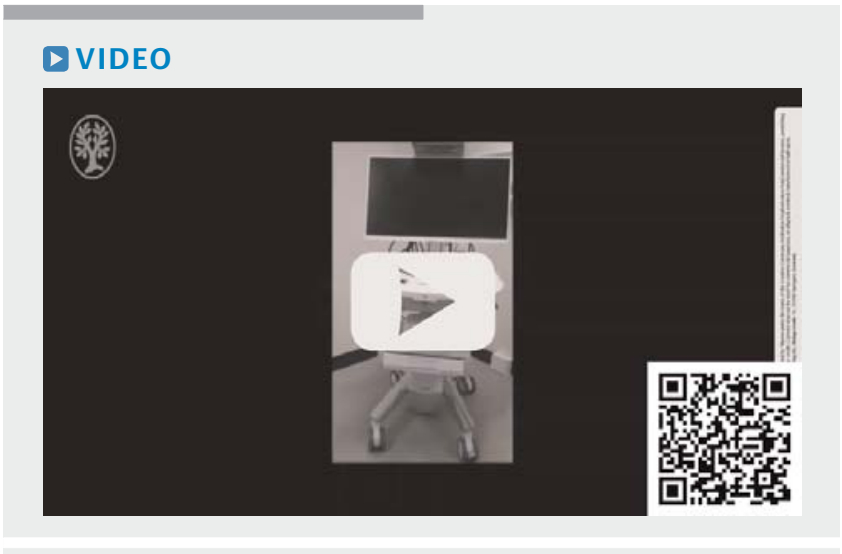

- Video 1 Example endoscopic video of C-POEM.

v2015b). Descriptive baseline characteristics were presented as mean with standard deviation (SD). Two-tailed paired t-test was used determine within-subject changes in patient-reported outcomes (SSQ and SWAL-QOL), HRPIM, VFSS, and distensibility metrics after C-POEM. Statistical analysis was performed in PRISM (GraphPad Software, LLC, USA, v8.4.2) and $P<0.05$ was considered statistically significant.

\section{Results}

\section{Baseline characteristics and clinical outcomes}

The prevalence of dysphagia symptom among consecutively screened 48 PD patients was $66.7 \%$. Of the dysphagic PD patients, $34.4 \%(11 / 32)$ met the manometric criteria for UES dysfunction and were offered C-POEM; 1 declined C-POEM and two were excluded (one had unacceptable anesthetic risk; one had deep-brain stimulator contraindicating monopolar diathermy). The mean age of the remaining eight patients undergoing CPOEM was 70 years $(S D=7.7)$ and four $(50 \%)$ were female. PD staging using Movement Disorder Society-sponsored revision of the United Parkinson's Disease Rating Scale (MDS-UPDRS) $[25,26]$ confirmed $100 \%(8 / 8)$ and $87.5 \%(7 / 8)$ of the patients had moderate-to-severe motor symptoms and signs, respectively ( $>$ Table 1 ). There was no achalasia of cardia.

C-POEM was technically feasible in all eight patients. The mean procedural time, defined as the duration from submucosal bleb injection to mucosotomy closure, minus the duration for CP distensibility measurement, was 66 minutes $(S D=23$ ). During the submucosal tunnel development, cervical subcutaneous emphysema was noted in all patients. However, there were no ventilatory difficulties, and the emphysema was largely resolved by the end of the procedure. Patients resumed oral medications with sips of water on the same day after recovery. A soft diet was introduced after overnight observation. Mild to moderate throat pain up to one week was managed using analgesia as required. All patients were discharged after 24-48 hours observation. No perforation, infection, or delayed bleeding was recorded.

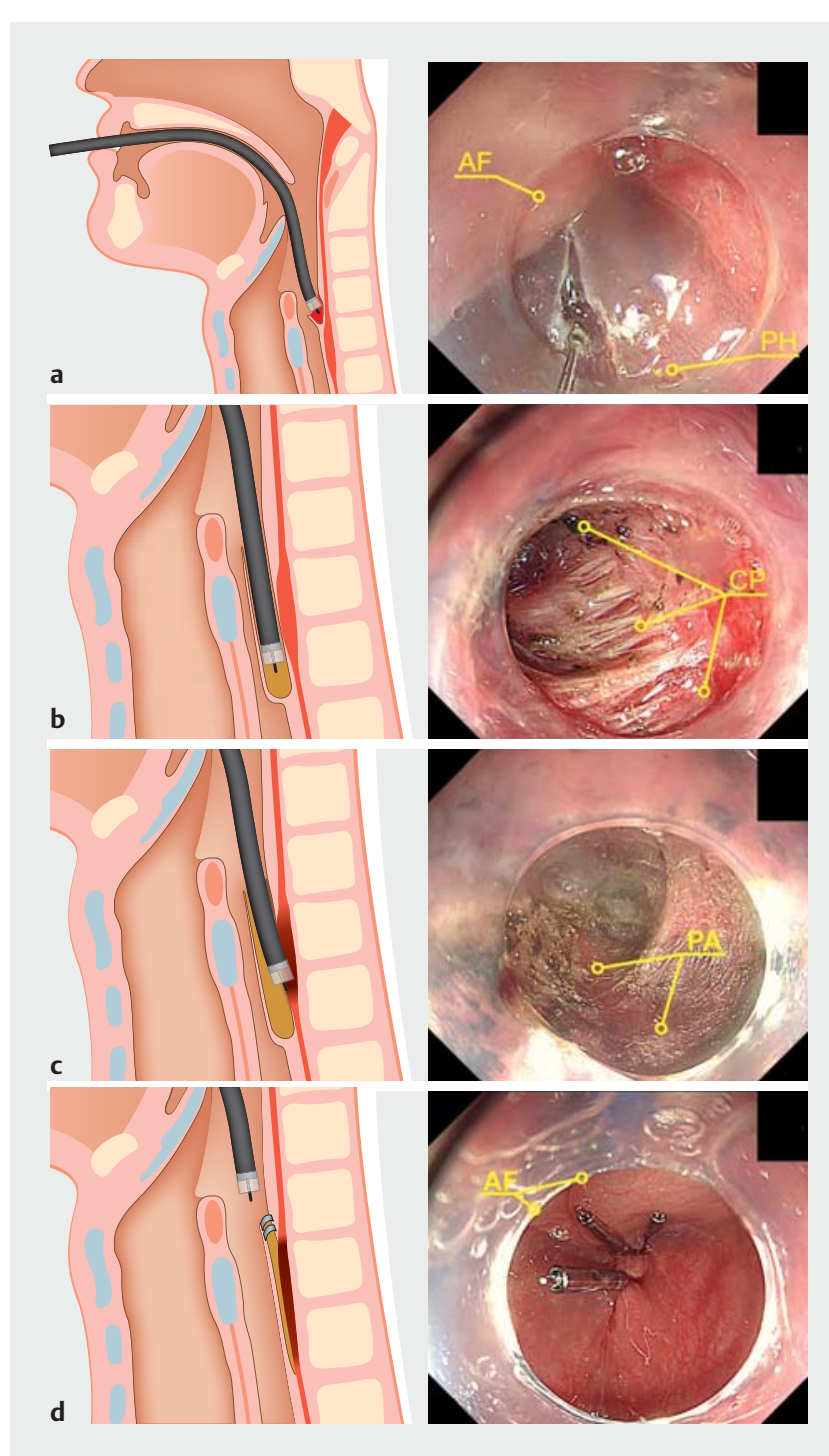

- Fig. 2 Schematic and endoscopic images during C-POEM. a Submucosal bleb and mucosotomy from the posterior hypopharyngeal wall (PH). Note the incision level is just below the aryepiglottic folds (AF). b Submucosal tunneling to proximal cervical esophagus and exposure of the $\mathrm{CP}$ horizontal fibers. $\mathbf{c}$ Full-thickness CP myotomy exposing the posterior adventitia (PA). d Closure of the mucosotomy using endoscopic clips. (Source left images: Sajith Udurawana)

At 1-month follow-up, seven patients (87.5\%) reported appreciable improvement in swallowing function during subjective, global assessment. SSQ score dropped (improved) significantly by an average of $45 \%$ from 621.5 to 341.8 (mean difference $-277.3,95 \% \mathrm{Cl}[-497.8,-56.7], \mathrm{t}(5)=2.97 \quad P=0.02$ ) ( $>$ Fig.3a). Similarly, SWAL-QOL score increased (improved) significantly by an average of $21.5 \%$ from 54.9 to 68.3 (mean difference $9.1,95 \% \mathrm{Cl}[0.7,17.5], \mathrm{t}(5)=2.57, P=0.037)$.

Longer-term follow-up showed the improvement in SSQ and SWAL-QOL scores were sustained in some patients ( $\mathbf{F i g . 3 b}$ ). Mixed-effect regression analysis showed a non-significant average increase in SSQ by 16 points per month $(P=0.082$, $95 \% \mathrm{Cl}[-2.0,34.5])$ and a non-significant average decrease in 
- Table 1 Baseline characteristics and MDS-UPDRS staging of patients.

\begin{tabular}{|c|c|c|c|c|c|c|c|c|c|c|}
\hline \multirow[b]{2}{*}{$\begin{array}{l}\text { Patient } \\
\text { no. }\end{array}$} & \multirow[b]{2}{*}{ Gender } & \multirow[b]{2}{*}{ Age } & \multicolumn{2}{|c|}{ Baseline } & \multirow{2}{*}{$\begin{array}{l}\text { Hoehn } \\
\text { and Yahr } \\
\text { Staging }\end{array}$} & \multicolumn{5}{|c|}{ MDS-UPDRS staging } \\
\hline & & & SSQ & $\begin{array}{l}\text { SWAL- } \\
\text { QOL }\end{array}$ & & $\begin{array}{l}\text { Non-motor } \\
\text { symptoms }\end{array}$ & $\begin{array}{l}\text { Motor } \\
\text { symptoms }\end{array}$ & $\begin{array}{l}\text { Motor } \\
\text { signs }\end{array}$ & $\begin{array}{l}\text { Motor com- } \\
\text { plications }\end{array}$ & Total \\
\hline 1 & $\mathrm{~F}$ & 69 & 422 & 40.5 & 3 & 12 & 25 & 52 & 4 & 93 \\
\hline 2 & $\mathrm{M}$ & 67 & 614 & 57.7 & 2 & 3 & 15 & 38 & 2 & 58 \\
\hline 3 & $\mathrm{~F}$ & 74 & 1034 & 62.9 & 3 & 17 & 23 & 66 & 0 & 106 \\
\hline 4 & $\mathrm{M}$ & 76 & 413 & 58.5 & 4 & 8 & 33 & 45 & 0 & 86 \\
\hline 5 & $M$ & 65 & 302 & 77.8 & 3 & 13 & 18 & 36 & 12 & 79 \\
\hline 6 & $\mathrm{~F}$ & 55 & 877 & 52.1 & 3 & 0 & 13 & 38 & 0 & 51 \\
\hline 7 & $\mathrm{~F}$ & 79 & 623 & 65.9 & 5 & 12 & 33 & 73 & 0 & 118 \\
\hline 8 & $M$ & 75 & 687 & 47.6 & 3 & 21 & 26 & 28 & 7 & 82 \\
\hline
\end{tabular}

MDS-UPDRS, Movement Disorder Society-sponsored revision of the United Parkinson's Disease Rating Scale.

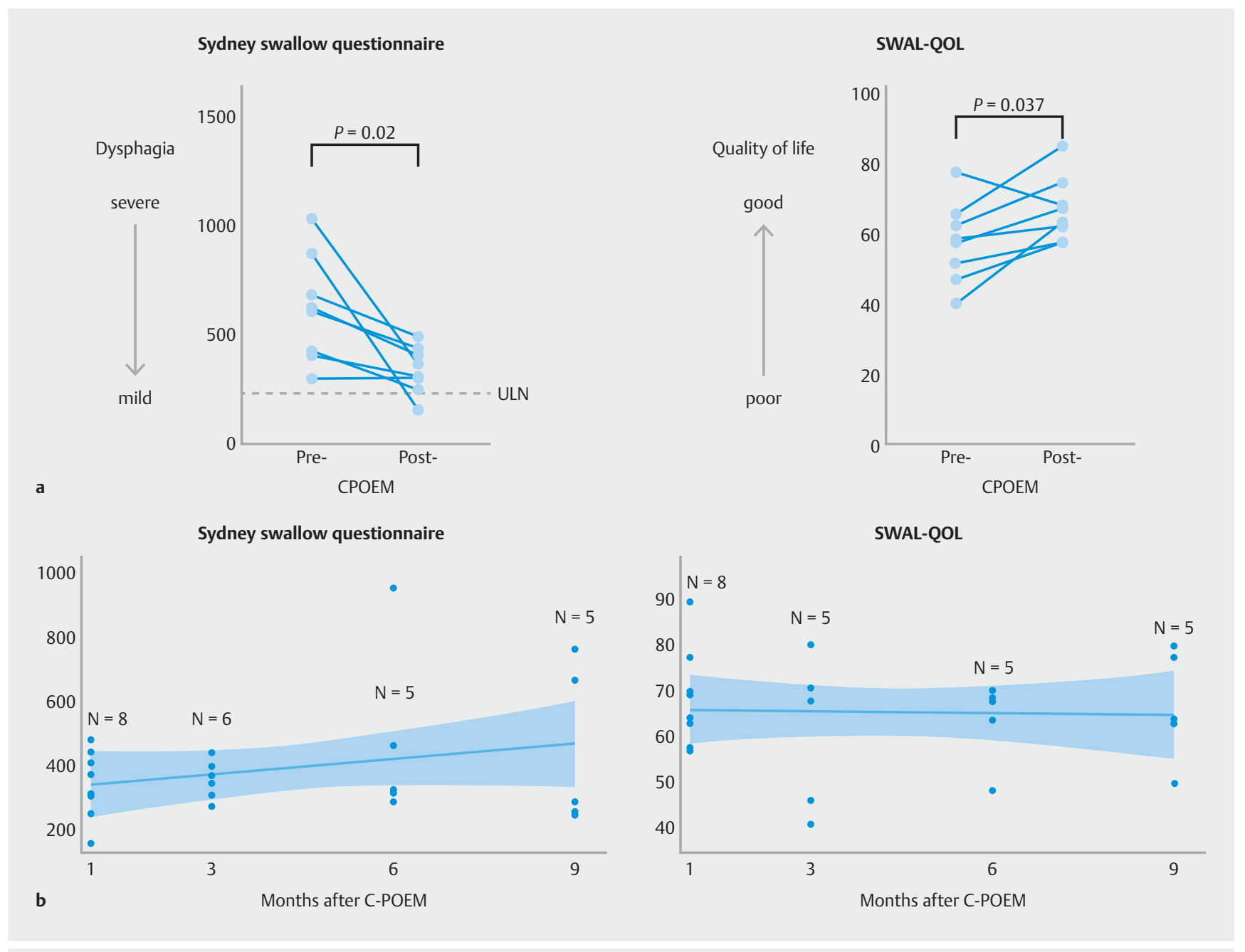

Fig. 3 a Patient-reported outcomes at 1 month after C-POEM and b longer-term follow-up. 

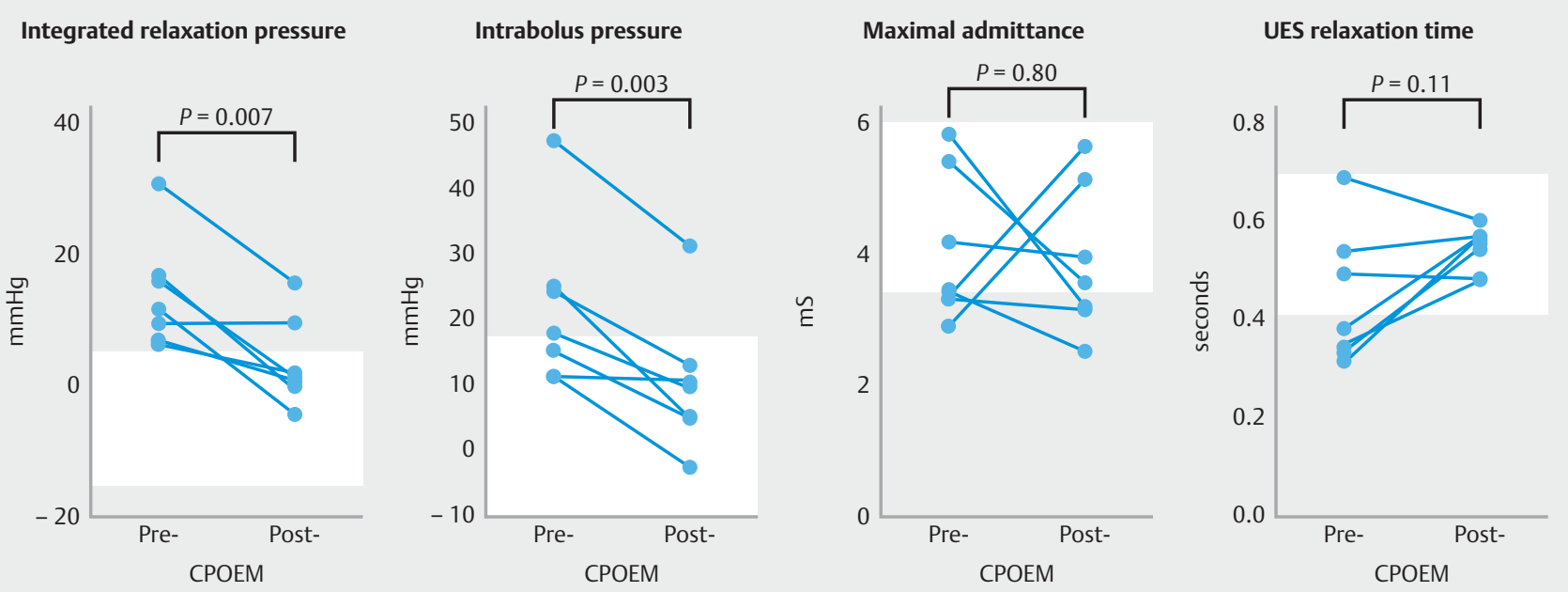

$5^{\text {th }}$ and $95^{\text {th }}$ percentile normative range

- Fig. 4 HRPIM UES metrics before and after C-POEM.

SWAL-QOL by 0.2 points per month $(P=0.77,95 \% \mathrm{Cl}[-1.3$, $0.9]$ ) by 9 -month follow-up.

\section{HRPIM}

Repeat HRPIM was performed in seven patients $(87.5 \%$ ) 1 month after C-POEM. Following myotomy, there was a significant decrease in the UES IRP from a mean of $13.7 \mathrm{~mm} \mathrm{Hg}$ to $3.6 \mathrm{~mm} \mathrm{Hg}$ (mean difference $-10.1 \mathrm{~mm} \mathrm{Hg}, 95 \% \mathrm{Cl}[-16.3$, -3.9], $\mathrm{t}(5)=3.98, P=0.007$ ) ( $\mathbf{F i g . 4}$ ). Five of seven demonstrated a return of post-C-POEM IRP values to within the normal range. Similarly, hypopharyngeal IBP decreased significantly from a mean of $23.5 \mathrm{~mm} \mathrm{Hg}$ to $10.4 \mathrm{~mm} \mathrm{Hg}$ (mean difference $-11.3 \mathrm{~mm} \mathrm{Hg}, 95 \% \mathrm{Cl}[-17.2,-5.4], \mathrm{t}(5)=4.7, P=0.003)$. The UES MaxAd remained unchanged with an average of $4.1 \mathrm{mS}$ and $3.9 \mathrm{mS}$ (mean difference $-0.2 \mathrm{mS}, 95 \% \mathrm{Cl}[-1.9,1.5], \mathrm{t}(5)=$ $0.27, P=0.8$ ), pre- and post-myotomy, respectively. UES RT was not impacted significantly by the procedure $(0.44$ seconds before to 0.54 seconds after POEM (mean difference 0.1 seconds, $95 \% \mathrm{Cl}[-0.03,0.22], \mathrm{t}(5)=1.87, P=0.11)$ ( Fig. 4 ).

\section{VFSS}

Before C-POEM, none of the patients had frank aspiration during VFSS, and none developed aspiration after C-POEM. Repeat VFSS was performed in 6 (75\%) patients 1 month after C-POEM. After myotomy, there was a significant increase in both the mean UES maximal opening diameter $(3.5 \mathrm{~mm}$ to $6.2 \mathrm{~mm}$, mean difference $2.7 \mathrm{~mm}, 95 \% \mathrm{Cl}[0.2,5.2], \mathrm{t}(5)=2.75, P=0.04)$ and opening duration ( $0.43 \mathrm{~s}$ to $0.56 \mathrm{~s}$, mean difference $0.13 \mathrm{~s}$ $95 \% \mathrm{Cl}[0.02,0.24], \mathrm{t}(5)=2.94, P=0.03)$ during a $10-\mathrm{mL}$ swallow ( $\triangleright$ Fig.5). Similarly, the AUC (a product of opening diameter and duration) increased from $1.07 \mathrm{~mm} . \mathrm{s}$ to $1.96 \mathrm{~mm} . \mathrm{s}$ (mean difference $0.88 \mathrm{~mm} . \mathrm{s}, 95 \% \mathrm{Cl}[0.0 .29,1.47]$, t(5) $=3.84$, $P=0.012)$.

\section{$\mathrm{CP}$ passive distensibility}

The mean nCSA at $40-\mathrm{mL}$ distension was unchanged after CPOEM (from $152.9 \mathrm{~mm}^{2}$ to $169.0 \mathrm{~mm}^{2}$, mean difference $\left.16.1 \mathrm{~mm}^{2}, 95 \% \mathrm{Cl}[-12.3,44.5], \mathrm{t}(5)=1.58, P=0.19\right)$. Similarly, the mean CP-DI at $40 \mathrm{ml}$ distension was unchanged after CPOEM (from $3.0 \mathrm{~mm}^{2} / \mathrm{mm} \mathrm{Hg}$ to $2.6 \mathrm{~mm}^{2} / \mathrm{mm} \mathrm{Hg}$, mean difference $\left.-0.3 \mathrm{~mm}^{2} / \mathrm{mm} \mathrm{Hg} 95 \% \mathrm{Cl}[-0.8,0.13], P=0.12\right)$.

\section{Discussion}

To our knowledge, this is the first demonstration of the feasibility of this novel application of the POEM endoscopic technique in the treatment of pharyngeal dysphagia attributable to UES dysfunction in PD. Within the limit of a small sample size, CPOEM induces objectively measured: 1 ) improvement in UES relaxation and trans-sphincteric resistance to bolus flow; 2 ) improvement in both UES maximal opening diameter and duration of UES opening; and 3) improvement in dysphagia severity and swallow-related quality of life.

The advent of HRPIM permits quantitative analysis of UES function [21]. In lieu of a pharyngeal equivalent to the Chicago esophageal HRM classification [27], we selected patients for C-POEM using stringent manometric criteria based on statistically-defined laboratory normative data. We defined UES IRP $>2.9 \mathrm{~mm} \mathrm{Hg}$ (95 $5^{\text {th }}$ percentile) as the main diagnostic criterion for incomplete UES relaxation [22]. Furthermore, patients had to demonstrate at least one additional abnormal UES metric: either increased flow resistance (i.e. IBP), or reduced UES opening (defined by either abnormal UES Maximal Admittance, or reduced UES RT), to be eligible for C-POEM. While it remains unclear whether such stringent criteria are required to select those who will benefit from C-POEM, we chose these rigorous, conservative criteria to ensure that all cases formed a homogeneous cohort with unequivocal incomplete UES relaxation and to reduce the risk unnecessary procedures. All 

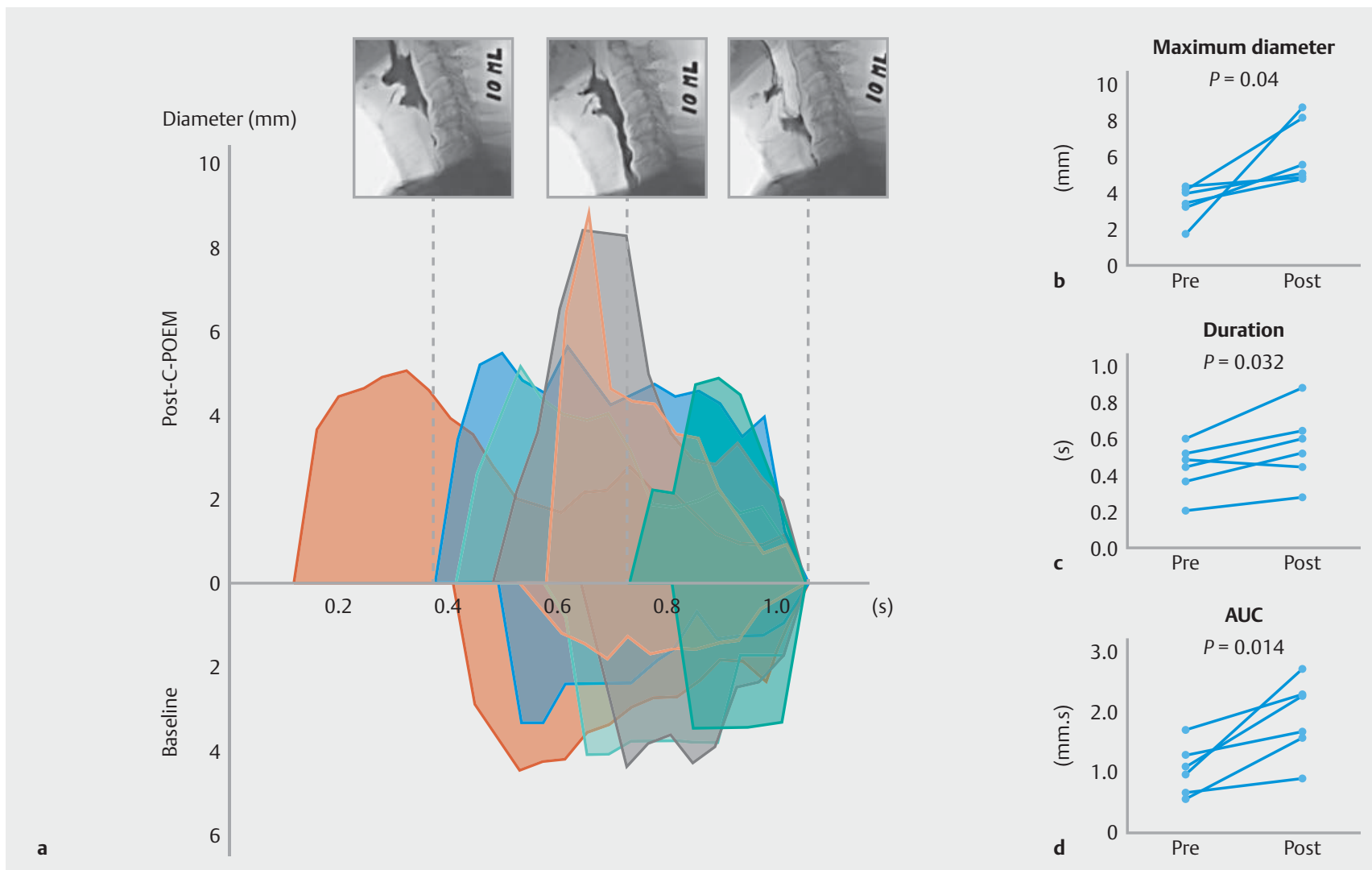

- Fig. 5 a Opening diameter of the UES measured on VFSS of a 10-mL bolus swallow in six individual patients at baseline and after C-POEM. For clarity UES opening has been synchronized to UES closure. Within-subject changes of $\mathbf{b}$ maximum UES opening diameter, $\mathbf{c}$ opening duration, and $\mathbf{d}$ AUC.

eight cases had subnormal UES opening confirmed on VFSS ( Fig.5) when compared to historical control data $[6,28]$ while intraprocedural distensibility measures did not change. These combined, objective, observations are consistent with the notion that the reduced UES opening in the conscious patient was not a consequence of a fibrotic structuring of the $\mathrm{CP}$, but rather centrally-mediated, neurogenic, incomplete UES relaxation.

Within the limit of a small sample size, our preliminary efficacy data are encouraging. Both patient-reported swallowing assessments, SSQ and SWAL-QOL improved following C-POEM. The difference in the potential therapeutic gain in swallowing function between (esophageal) POEM and C-POEM needs to be considered. In esophageal achalasia, retrosternal hold-up and regurgitation are almost entirely attributable to impaired trans-sphincteric flow. Thus, myotomy to the lower esophageal sphincter permits gravity to effectively clear the aperistaltic esophagus, potentially completely resolving all dysphagia symptoms [29]. In contrast, in Parkinson's, incomplete UES relaxation is only one of several vital biomechanisms underpinning dysphagia. In the context of manometrically proven failed UES relaxation in PD [6], or in the context of brain stem lesions [30], the ultimate severity of dysphagia correlates with the associated reduction of pharyngeal propulsion. Of course, disturbed oral preparation or oral delivery [31], will not respond to the CP myotomy. These considerations explain in part our findings that complete resolution of dysphagia symptoms (i. e., normalization of the SSQ scores) following C-POEM was not observed. Not knowing the extent to which C-POEM would benefit dysphagia resulting from a complex pathophysiology, we included a QoL assessment in the study design. SWAL-QOL provides a holistic assessment, including psychological and social consequences of dysphagia [32]. Our data suggests C-POEM induces at least a modest improvement dysphagia-specific QoL. Further studies are required to determine the extent to which C-POEM could restore acceptable swallow function and QoL, the relative impact of disturbed pharyngeal propulsive forces, and whether C-POEM might mitigate the risk of aspirationrelated events. Such data is required to define more precisely the population of Parkinsonian patients that are likely to benefit from C-POEM.

An important strength of the currently study is the diversity of objective measurement tools used to evaluate the oropharyngeal function. Repeat HRPIM after C-POEM confirmed significant reduction in UES relaxation pressure (IRP) and resistance to flow (IBP), confirming the myotomy targeted the intended anatomical structure. VFSS suggests an average increase in the maximal UES opening diameter by $2.7 \mathrm{~mm}$ after C-POEM, but similar improvement was not observed in the MaxAd, an impedance-based HRPIM metric that serves as a surrogate marker 
for maximum UES opening [21,33]. Our sample size is probably too small to determine the exact explanation for this conflicting data. Of interest, VFSS confirmed an increase in the UES opening duration and RT. As bolus flow is affected by both the duration permissive to flow and the maximal luminal diameter, the interplay between these two sphincter characteristics, and more importantly, how it is influenced by C-POEM, would be a focus of future studies.

Intraprocedural FLIP-measured intrinsic CP distensibility was not altered by myotomy. Superficially this seems counterintuitive, given previous studies have confirmed FLIP to be sensitive in demonstrating improved CP distensibility following simple mechanical dilatation or septotomy $[23,24]$. However, the fundamental difference lies in the fact that those studies examined patients with fibrosis-related structural pathologies (post-chemoradiotherapy stricture and Zenker's diverticulum) demonstrating restricted opening of normally relaxing UES [23, 24]. In contrast the cohort in the present study had incomplete (neurogenic) relaxation of a normally compliant UES in its passive state.

There are several important procedural considerations specific to the C-POEM. In our experience, the most challenging part of the C-POEM was the initial submucosal tunnel development because the mucosotomy and the initial trimming are both performed in the hypopharynx; the presence of the laryngeal structures (many are cartilaginous) and the endotracheal tube significantly restricts the scope movement into the submucosal plane. Furthermore, unlike the Zenker's diverticulum, there are no 'landmark' structural abnormalities (i.e., a septum) to guide the mucosotomy during C-POEM. We performed the mucosotomy in the posterior hypopharyngeal at a level (cusp of aryepiglottic folds) that would minimize the risk of clip aspiration. The restriction in the highest possible mucosotomy extent means the tunnel is invariably short $(1-2 \mathrm{~cm})$ before reaching the $\mathrm{CP}$ level, thereby further increasing the procedural difficulties.

Surgical emphysema in the neck and face was observed in all patients intra-procedurally; however, this did not adversely affect the ventilation, and the emphysema was largely resolved by the end of the procedure due to the rapid absorbability of carbon dioxide. The hypopharyngeal mucosotomy and clips were surprisingly well-tolerated requiring minimal analgesia. Similarly, inadvertent clip aspiration was not observed despite the concern.

VFSS was not performed after C-POEM to exclude leakage. Indeed, we allowed oral medication privilege immediately after recovery to avoid exacerbation of PD-related motor symptoms. The reason for omitting the VFSS was the lack of a suitable contrast media. Gastrografin is the conventional contrast of choice to exclude perforation; however, the aspiration risk in PD patients makes Gastrografin unsuitable for the purpose. A potential alternative agent is iohexol (Omnipaque); however, its safety in events of aspiration had also been questioned [34].

In the current study, we only recruited PD patients for CPOEM to minimize heterogeneity. There exists a spectrum of neuromyogenic disorders other than PD that can manifest UES dysfunction via injuries to the swallow pattern generator in the medulla (e. g. lateral medullary syndrome and other vascular lesions, tumor). Thus, the potential utility of C-POEM in the management of such disorders need to be considered in future studies.

\section{Conclusions}

In conclusion, the current study confirmed feasibility and safety of C-POEM as a treatment for dysphagia attributable to UES dysfunction in PD patients. Preliminary efficacy data was tantalizing for further studies to determine the extent of potential for C-POEM to benefit neuromyogenic OPD, and equally importantly, to determine how best to select patients who will most likely benefit from this procedure.

\section{Competing interests}

Dr. Omari holds inventorship of the patent family that covers the analytical methods deployed to the swallowgateway.com website, which is owned and provided by Flinders University.

Clinical trial

Australian New Zealand Clinical Trial Registry ACTRN12619000927123

TRIAL REGISTRATION: Non-randomized Interventional Trial ACTRN12619000927123 at https://www.anzctr.org.au/

References

[1] Kalia L, Lang A. Parkinson's disease. Lancet 2015; 386: 896-912

[2] Pringsheim T, Jette N, Frolkis A et al. The prevalence of Parkinson's disease: A systematic review and meta-analysis. Movement Disorders 2014; 29: 1583-1590

[3] Dorsey ER, Constantinescu R, Thompson JP. Projected number of people with Parkinson disease in the most populous nations, 2005 through 2030. Neurology 2007 2007; 68: 384-386

[4] Suttrup I, Warnecke T. Dysphagia in Parkinson's Disease. Dysphagia 2016; 31: 24-32

[5] Menezes C, Melo A. Does levodopa improve swallowing dysfunction in Parkinson's disease patients? J Clin Pharm Ther 2009; 34: 673-676

[6] Ali GN, Wallace KL, Schwartz R et al. Mechanisms of oral-pharyngeal dysphagia in patients with Parkinson's disease. Gastroenterology 1996; 110: 383-392

[7] Alfonsi E, Versino M, Merlo IM et al. Electrophysiologic patterns of oral-pharyngeal swallowing in parkinsonian syndromes. Neurology 2007; 68: 583-589

[8] Knigge MA, Thibeault SL. Swallowing outcomes after cricopharyngeal myotomy: A systematic review. Head Neck 2018; 40: 203-212

[9] Cook IJ, Kahrilas PJ. AGA technical review on management of oropharyngeal dysphagia. Gastroenterology 1999; 116: 455-478

[10] Born L], Harned RH, Rikkers LF. Cricopharyngeal dysfunction in Parkinson's disease: role in dysphagia and response to myotomy. Movement Disorders 1996; 11: 53-58

[11] Brigand C, Ferraro P, Martin J et al. Risk factors in patients undergoing cricopharyngeal myotomy. Br J Surg 2007; 94: 978-983 
[12] Inoue $\mathrm{H}$, Minami H, Kobayashi $\mathrm{Y}$ et al. Peroral endoscopic myotomy (POEM) for esophageal achalasia. Endoscopy 2010; 42: 265-271

[13] Jacobs CC, Perbtani Y, Yang D et al. Per-oral endoscopic myotomy for esophagogastric junction outflow obstruction: a multicenter pilot study. Clin Gastroenterol Hepatol 2021; 19: 1717-1719 doi:10.1016/ j.cgh.2020.08.048

[14] Khashab MA, Stein E, Clarke JO et al. Gastric peroral endoscopic myotomy for refractory gastroparesis: first human endoscopic pyloromyotomy (with video). Gastrointest Endosc 2013; 78: 764-768

[15] Wahba G, Bouin M. Jackhammer esophagus: A meta-analysis of patient demographics, disease presentation, high-resolution manometry data, and treatment outcomes. Neurogastroenterol Motil 2020; 32: e13870

[16] Yang JN, Novak S, Ujiki M et al. An international study on the use of peroral endoscopic myotomy in the management of Zenker's diverticulum. Gastrointest Endosc 2020; 91: 163-168

[17] Wallace KL, Middleton S, Cook IJ. Development and validation of a self-report symptom inventory to assess the severity of oral-pharyngeal dysphagia. Gastroenterology 2000; 118: 678-687

[18] Szczesniak MM, Maclean J, Zhang T et al. The normative range for and age and gender effects on the Sydney Swallow Questionnaire (SSQ). Dysphagia 2014; 29: 535-538

[19] Plowman-Prine EK, Sapienza CM, Okun MS et al. The relationship between quality of life and swallowing in Parkinson's disease. Mov Disord 2009; 24: 1352-1358

[20] McHorney CA, Robbins J, Lomax K et al. The SWAL-QOL and SWALCARE outcomes tool for oropharyngeal dysphagia in adults: III. Documentation of reliability and validity. Dysphagia 2002; 17: 97-114

[21] Omari TI, Ciucci M, Gozdzikowska K et al. High-resolution pharyngeal manometry and impedance: protocols and metrics-recommendations of a high-resolution pharyngeal manometry international working group. Dysphagia 2020; 35: 281-395

[22] Ferris L, Doeltgen S, Cock C et al. Modulation of pharyngeal swallowing by bolus volume and viscosity. Am J Physiol Gastrointest Liver Physiol 2021; 320: G43-G53
[23] Zhang L, Wu P, Szczesniak M et al. Clinical utility of cricopharyngeal distensibility measurements during endoscopic myotomy for Zenker's diverticulum. Gastrointest Endosc 2021; 93: 390-397

[24] Wu P, Szczesniak MM, Maclean J et al. Clinical utility of functional imaging probe in management of dysphagia following head and neck cancer therapies. Endoscopy 2017; 49: 1-8

[25] Goetz CG, Tilley BC, Shaftman SR et al. Movement Disorder Societysponsored revision of the Unified Parkinson's Disease Rating Scale (MDS-UPDRS): scale presentation and clinimetric testing results. Mov Disord 2008; 23: 2129-2170

[26] Martinez-Martin P, Rodriguez-Blazquez C, Mario A et al. Parkinson's disease severity levels and MDS-Unified Parkinson's Disease Rating Scale. Parkinsonism Relat Disord 2015; 21: 50-54

[27] Yadlapati R, Kahrilas PJ, Fox MR et al. Esophageal motility disorders on high-resolution manometry: Chicago classification version 4.0๑. Neurogastroenterol Motility 2021; 33: e14058

[28] Jacob P, Kahrilas P, Logemann J et al. Upper esophageal sphincter opening and modulation during swallowing. Gastroenterology 1989; 97: $1469-1478$

[29] Katzka DA, Camilleri M. Treating the pylorus in gastroparesis: The new riddle wrapped in the ultimate enigma? Gastrointest Endosc 2020; 91: $1300-1302$

[30] Williams RB, Wallace KL, Ali GN et al. Biomechanics of failed deglutitive upper esophageal sphincter (UES) relaxation in patients with neurogenic dysphagia. Am J Physiol 2002; 283: G16-G26

[31] Simons JA. Swallowing dysfunctions in Parkinson's disease. Int Rev Neurobiol 2017; 134: 1207-1238

[32] Keage M, Corben L, Vogel A. A systematic review of self-reported swallowing assessments in progressive neurological disorders. Dysphagia 2015; 31: 27-46

[33] Omari TI, Ferris L, Dejaeger E et al. Upper esophageal sphincter impedance as a marker of sphincter opening diameter. Am J Physiol Gastrointest Liver Physiol 2012; 302: G909-G913

[34] Bopeththa BVKM, Hewavithana PB, Hewapathirana HLI et al. Myasthenic crisis following iodinated contrast material (iohexol) aspiration: a case report. J Med Case Rep 2019; 13: 166 doi:10.1186/ s13256-019-2114-8 